\title{
The Impact of Measuring Patient-Reported Outcome Measures on Quality of and Access to Palliative Care
}

\author{
Deborah Dudgeon, MD, FRCPC
}

\begin{abstract}
Background: Measuring performance for palliative care is complex as care is delivered in many sites, over time and jointly to the patient and family. Measures of structural processes do not necessarily capture aspects that are important to patients and families nor reflect holistic multidisciplinary outcomes of care. This article focuses on the question as to whether measurement of patient-reported outcome measures improves the outcomes of quality and access to palliative care.

Objectives: To review the international evidence that measurement of indicators of desired outcomes improves the quality of and access to palliative care, in order to apply them to the Canadian context.

Design: Rapid review.

Setting: Canadian context.

Findings: This review identified six systematic reviews and forty-seven studies that describe largely national efforts to arrive at a consensus as to what needs to be measured to assess quality of palliative care. Patientreported outcome measures (PROMs) are becoming more prevalent, with emerging evidence to suggest that their measurement improves outcomes that are important to patients. Several Canadian initiatives are in place, including the Canadian Partnership Against Cancer's efforts, in conjunction with other partners, to develop common quality measures. Results from Australia's Palliative Care Outcomes Collaborative demonstrate that patient-centered improvements in palliative care can be measured by using patient-reported outcomes derived at the point of care and delivered nationally.

Conclusions: Measurement of quality palliative and end-of-life care is very complex. It requires that both administrative data and PROMs be assessed to reflect outcomes that are important to patients and families. Australia's national initiative is a promising exemplar for continued work in this area.
\end{abstract}

Keywords: indicators; palliative care; patient-reported outcome measures; quality assessment

\section{Introduction}

$\mathbf{I}$

F WE WANT TO MANAGE the system for improvement, then we need to measure where things are, what is getting better, and what is not. Measuring performance for palliative care is complex as care is delivered at a number of sites, including the home, clinic, hospital, long-term care facilities, and acute care. As well, measures must capture aspects that are important to patients and families and reflect holistic multidisciplinary outcomes of care. ${ }^{1}$ The Institute of Medicine's report "Crossing the Quality Chasm" outlined the aims of the healthcare system consistent with these aims: to deliver care that is safe, effective, patient centered, timely, efficient, and equitable. ${ }^{2}$
Palliative Care Matters assessed those aspects that were important to the public, including patients and their families. The IPSOS survey showed that the majority of Canadians thought that surveys should be conducted among family members $(84 \%)$, caregivers $(82 \%)$, and patients $(79 \%)$ regarding the care received, and $72 \%$ of Canadians agreed that annual surveys should be conducted to measure and track Canadians' awareness levels of palliative care (Roulston, this issue).

This article will:

1. Outline the components and ways of measuring quality; 
2. The evidence that measuring patient-reported and other outcomes improves the quality of palliative care; and

3. Canadian efforts to measure structure, process, and outcomes related to palliative care.

\section{Methods}

A systematic identification and retrieval of articles was conducted according to the methods outlined by Sims and Fassbender in this issue. Articles were included if they provided evidence related to outcomes associated with palliative care. Second, articles required that indicators were included, regardless of the methods used to ascertain consensus. Articles that specifically addressed how, or if, measuring patientreported and other outcomes improved the quality of palliative care were identified. A narrative review, synthesis, and contextualization within Canada follows.

\section{Findings and Discussion}

\section{Measuring quality}

Donabedian authored the most widely adopted framework used to assess quality. He proposed three components as required to evaluate the quality of healthcare: structure, process, and outcomes. ${ }^{3,4}$ These components have since been widely adopted in the assessment of quality, although with a preponderance of assessing the "easier" domains of structure and process. Assessment of structure addresses the question "which resources are required/used," including settings, qualifications of providers, and administrative systems that provide the service. Process evaluation addresses "how are the resources used" and the components of care delivered.

Outcome evaluation answers "what have we achieved." It focuses on the change in the person's health status (recovery, restoration, and survival) and quality of life that can be attributed to the healthcare received. ${ }^{4-7}$ Donabedian emphasized the need for reliable measures of structure and process that could be linked to outcomes so that quality improvements could be made. Ultimately, the outcome is the most important as it directly affects the patient and family, assesses the effectiveness of interventions, and encompasses the results of the whole cycle of care. Improved outcomes in healthcare can reduce the burden of the illness on the individual and family, and/or society.

\section{Outcome measures}

Outcome measures are used in palliative care for clinical, audit, and research purposes. ${ }^{8}$ In clinical care, outcome measures are used to: monitor symptom intensity, functional status, and quality of life; aid in decision making; facilitate communication between team members and patients/ families; and evaluate effectiveness of treatments. Audit involves setting standards for desired outcomes, monitoring practices, and evaluating performance of individual programs, regions, and/or countries. Outcome measurement that is critical for research sets out to demonstrate results of interventions and evaluate the quality of care.

Patient and family outcomes, in particular, are often measured by using questionnaires, which are called patientreported outcome measures (PROMs). A PROM allows people to identify their baseline and changes in their health and functional status, quality of life, symptoms, and satisfaction with care. Studies that examined what patients and families value most at the end of life found that these include:

- adequate pain and symptom management,

- psychosocial care,

- good communication of information,

- clear decision making,

- avoiding prolonged dying,

- preparing for death,

- dying in the preferred place,

- avoidance of aggressive care,

- sense of control,

- creating a sense of completion,

- contributing to others,

- strengthening relationships, and

- affirming the whole person. $6,9,10$

Outcomes also track performance at the system level and are generally measured by using administrative data. These outcomes include items such as: place of death (hospital or home), number of days in an acute care hospital in the last six months of life, and deaths in intensive care units and emergency room visits in the last two weeks of life. Such measures have value not only to individual patients, as most would like to live and die in their own homes, but also to society through potential resource and cost savings.

\section{Indicators}

Quality indicators are used to help measure the quality of care and services delivered. They are well defined and measure specific aspects of desired outcomes, processes, or structures of care. ${ }^{11}$ They are usually described with a numerator and denominator on an aggregated level such as number or percentage of patients (e.g., $70 \%$ of patients showed improvements). ${ }^{5,12}$ Quality indicators are effective and quick tools for assessing service performance at individual, program, regional, national, and international levels. Until recently, almost all quality assessments of palliative care focused on care structures and processes rather than outcomes. $^{8}$

The literature includes a number of systematic reviews that identify quality indicators and measures used in palliative care. ${ }^{12-17}$ A recent review of quality measures for palliative care in patients with cancer conducted by Kamal et al. identified 284 quality measures and 13 measure sets. Of these, $35 \%$ related to physical aspects, $23 \%$ related to structure and process, and only $4 \%$ related to spiritual and $1 \%$ cultural aspects.

Numerous international groups have attempted to systematically identify and agree on quality indicators. They have:

- identified indicators or performance measures that are relevant to hospice and palliative care;

- narrowed the list through a modified Delphi rating process through consultation with different professional and patient groups; and

- established consensus on a set of quality indicators for hospice and palliative care program service assessment. $^{18-24}$

Evidence that measuring improves quality of and access to palliative care 
Studies of routine collection of PROM in clinical practice demonstrate strong to very strong evidence of:

- a positive effect on patient-clinician communication, ${ }^{25,26}$

- identification of unrecognized symptoms, ${ }^{27,28}$

- increased monitoring of symptoms, ${ }^{25}$

- a larger number of actions taken based on quality-oflife data, ${ }^{28}$

- improved patient satisfaction and experience, ${ }^{25}$

- association between higher symptom scores and higher rates of clinical action, and ${ }^{29}$

- less reports of debilitating physical symptoms at a subsequent visit. $^{30}$

A recent systematic review found that there was moderate evidence that using PROMs and providing feedback on the information improved psychological and emotional factors for patients. The evidence did not show any improvement in symptom burden or overall quality of life. ${ }^{28}$ Another review found that interventions that focused on quality-of-life assessment were moderately effective. ${ }^{31}$ Most studies, however, examined elements of the processes of care rather than outcomes. $^{32,33}$

Kamal et al. investigated the relationship between qualitymeasure-based care and quality of life in a community-based palliative care consortium to determine which components of palliative care must be delivered consistently to achieve improved outcomes. ${ }^{34}$ They found that measures related to assessing emotional well-being and comprehensive symptom screening were significantly associated with the highest quality of life. They concluded that measuring these may improve patient outcomes. However, because there are so few studies, there is some uncertainty about the impact of routinely collected PROMs on quality improvement, public reporting, or system performance. ${ }^{25}$

In a pilot study of a quality improvement project, Abernethy et al. demonstrated that an intervention and patientreported symptom data collected with tablet computers had a positive effect on distress and despair for patients with advanced cancer and worsening symptoms. ${ }^{35}$ In another study, England hospices and community palliative care teams were able to use a set of quality indicators as a tool to help develop plans for quality improvement and to achieve almost all of their desired changes. ${ }^{36}$ A study by Barbera et al. found that women with breast cancer receiving adjuvant chemotherapy who routinely completed an Edmonton Symptom Assessment System (ESAS), a measure of symptom intensity, were less likely to present to the emergency department than those who did not. ${ }^{37}$

Australia has a national program funded by the federal Department of Health called the Palliative Care Outcomes Collaborative (PCOC). The program is designed to improve palliative care through an audit and feedback quality cycle. It is helping palliative care services measure the quality of symptom control and develop national benchmarks to understand specialized hospice and palliative care service outcomes. ${ }^{38}$ Services who join the PCOC agree to use common patient outcome measures at the point of care.

Essential characteristics of the PCOC include:

- national service-level performance data that are derived from the common outcome measures;
- national benchmark standards against which individual services compare themselves;

- nationally employed staff that support palliative care services to identify priorities for clinical and systems change and help implement quality improvement initiatives; and

- collection of aggregated data that are analyzed and reported back to each service every six months.

The PCOC analyzed data from January 2009 to December 2011 for eight of the routinely reported measures, including physical, psychological, and family/carer domains. The analysis showed there were statistically significant improvements in all domains of both patient- and clinicianreported outcomes at a palliative care service level except for pain. $^{39}$ This paper, published in 2015 , is the first and only one to demonstrate that patient-centered improvements in palliative care can be measured by using patient- and familycentered outcomes derived at the point of care and tracked nationally.

\section{Canadian context}

In 1995, the Special Senate Committee on Euthanasia and Assisted Suicide reported that accurate statistical data regarding numbers of programs, institutions, number of patients, comparative costs, and other aspects of palliative care were scarce. ${ }^{40}$ In the intervening years, strides have been made to understand palliative and end-of-life care through different regional, provincial/territorial, and national initiatives.

In 1996, Health Canada funded a pilot study to collate data from six palliative care programs located in five cities across Canada. ${ }^{41}$ In 2009, the Canadian Partnership Against Cancer (CPAC) funded a project to improve palliative care programs' electronic data sources to better understand the characteristics of terminally ill cancer patients and their resource use in the final year of life. The study included noncancer patients as a comparator group. ${ }^{42}$

Both the 1996 and 2009 Health Canada projects used program-level data to address structure and process questions. They found a variety of formats, software, and coding structures with variable completeness, accuracy, and consistency between the different programs. ${ }^{41,42}$ The 2009 project found that few programs collected electronic PROMs such as the ESAS or functional status and none collected quality-of-life or patient/family satisfaction data. ${ }^{42}$

Currently, most provinces and territories are developing palliative care indicators to help monitor service delivery. Administrative data have been used to examine process and outcome components of quality at a provincial level, including:

- service utilization patterns $^{43-45}$;

- the association between home palliative care services and end-of-life care indicators ${ }^{44}$ and costs ${ }^{46}$; and

- to make comparisons of health service quality indicators between three ${ }^{46}$ or four provinces ${ }^{37}$

At a national level, in 2013, the Canadian Institute for Health Information (CIHI) highlighted the variation in service use across provinces. It examined the utilization of hospital services in the last 28 days of life by cancer patients who died in acute care hospitals. $^{47}$ 
The CPAC produces an annual Cancer System Performance Report that provides a pan-Canadian cross-section of key performance indicators. In the 2016 report, two indicators related to palliative care were included: place of death and the number of provinces conducting standardized screening for distress in cancer centers, a process indicator related to PROMs. The report indicates that there was a threefold increase in the number of provinces reporting province-wide implementation of standardized screening for distress in cancer centers from 2007 to 2015, 2/10 and 7/10 respectively. ${ }^{48}$

The Partnership is also working with the National Palliative and End-of-Life Care Network and other partners to:

- develop common quality indicators and benchmarks;

- develop a common, systematic way to collect and report on PROMs across provinces; and

- report these data from across the country.

Currently, PROMs data are only available from cancer centers. However, Accreditation Canada standards include a recommendation that organizations administer ESAS on admission and track performance measures for hospice and palliative care clients, ${ }^{49}$ so data should be available to monitor performance and aggregate information.

\section{Conclusion}

Measurement of quality palliative and end-of-life care is very complex. It requires that both administrative data and PROMs be assessed to reflect outcomes that are important to patients and families.

Quality outcome measures, indicators, and necessary common data elements are available and have demonstrated value in improving outcomes and the quality of care. Although there are some reports in Canada since the Senate Committee's observation that data were scarce, there is currently no coordinated effort to collect common data elements at a palliative care program level.

Accreditation Canada has a standard for administration of ESAS, a PROM regarding symptom intensity, but these data are not collected and reported to allow for comparisons and improvements.

The CPACs initiative to increase standardized screening for distress for cancer patients across Canada has shown significant growth and could be expanded to include the noncancer palliative patient. As previously stated, CPAC is working with the National Palliative and End-of-Life Care Network and other partners to develop: common quality indicators and benchmarks; a common, systematic way to collect and report on PROMs across provinces and to report these data from across the country.

Australia's national initiative is an excellent model for Canada to explore. It has common patient-reported outcomes, national benchmark standards, and nationally employed staff to support palliative care services to identify priorities for quality improvement, coupled with collection of aggregated data and reporting back to the individual services.

\section{Acknowledgments}

The author acknowledges the contributions of the Palliative Care Matters Research Support Team: Sharna Polard and Roger Salus for library services and literature searching; Heather Stiles and Michelle Sims for overall research coordination and assistance; and Michelle Sims for literature screening, literature review, and project support.

\section{Author Disclosure Statement}

The author is the Senior Scientific Lead for the PersonCentered Perspective for the Canadian Partnership Against Cancer.

\section{References}

1. Higginson I, Simon S, Benalia H, et al.: Republished: Which questions of two commonly used multidimensional palliative care patient reported outcome measures are most useful? Results from the European and African PRISMA survey. Postgrad Med J 2012;88:451-457.

2. Committee on Quality of Health Care in America, Institute of Medicine Washington DC: Crossing the Quality CHasm: A New Health System for the 21st Century. Washington DC: Committee on Quality of Health Care in America, Institute of Medicine, 2001.

3. Ayanian J, Markel H: Donabedian's lasting framework for health care quality. N Engl J Med 2016;375:205-207.

4. Porter M, Larsson S, Lee T: Standardizing patient outcomes measurement. N Engl J Med 2016;374:504-506.

5. Bausewein C, Daveson B, Benalia H, et al.: Outcome Measurement in Palliative Care: The Essentials. London: King's College, 2015.

6. Capelas MLV, Vicuna MN, Rosa FC: Quality assessment in palliative care-An overview. Eur J Palliat Care 2013; 20:196-198.

7. Kamal A, Currow D, Ritchie C, et al.: The value of data collection within a palliative care program. Curr Oncol Rep 2011:13:308-315.

8. Bausewein C, Daveson B, Currow DC, et al.: EAPC White Paper on outcome measurement in palliative care: Improving practice, attaining outcomes and delivering quality services-Recommendations from the European Associan for Palliative Care (EAPC) Task Force on Outcome Measurement. Palliat Med 2016;30:6-22.

9. Steinhauser KE, Christakis NA, Clipp EC, et al.: Factors considered important at the end of life by patients, family, physicians and other care providers. JAMA 2000;284: 2476-2482.

10. Bainbridge D, Brazil K, Drueger P, et al.: A proposed systems approach to the evaluation of integrated palliative care. BMC Palliat Care 2010;9:1-12.

11. Mainz J: Defining and classifying clinical indicators for quality improvement. Int J Qual Health Care 2003;15:523-530.

12. De Roo M, Leemans K, Claessen S, et al.: Quality indicators for palliative care: Update of a systematic review. J Pain Symptom Manage 2013;46:556-572.

13. Raijmakers N, Galushko M, Domeisen F, et al.: Quality indicators for care of cancer patients in their last days of life: Literature update and experts' evaluation. J Palliat Med 2012;15:308-316.

14. Parker D, Hodgkinson B: A comparison of palliative care outcome measures used to assess the quality of palliative care provided in long-term facilities: A systematic review. Palliat Med 2011;25:5-20.

15. Ostgathe C, Voltz R: Quality indicators in end-of-life care. Curr Opin Support Palliat Care 2010;4:170-173.

16. Mularski R, Dy S, Shugarman L, et al.: A systematic review of measures of end-of-life care and its outcomes. Health Serv Res 2007;42:1848-1870. 
17. Kamal A, Gradison M, Maguire J, et al.: Quality measures for palliative care in patients with cancer: A systematic review. J Oncol Pract 2014;10:281-287.

18. Nakazawa Y, Kato M, Yoshida S, et al.: Population-based quality indicators for palliative care programs for cancer patients in Japan: A Delphi study. J Pain Symptom Manage 2016;51:652-661.

19. Woitha K, Van Beek K, Ahmed N, et al.: Development of a set of process and structure indicators for palliative care: The Europall project. BMC Health Serv Res 2012;12:381.

20. Dy SM, Kiley K, Ast K, et al.: Measuring what matters: Top-ranked quality indicators for hospice and palliative care from the American Academy of Hospice and Palliative Medicine and Hospice and Palliative Nurses Association. J Pain Symptom Manage 2015;49:773-781.

21. National Quality Forum: Palliative Care and End-of-Life Care Measures. Washington DC: National Quality Forum, 2012.

22. Leemans K, Deleins L, Van den Block L, et al.: Systematic quality monitoring for specialized palliative care services: Development of a minimal set of Quality Indicators for Palliative Care study (QPAC). Am J Hosp Palliat Med 2017;34:532-546.

23. Woitha K, Van Beek K, Ahmed N, et al.: Validation of quality indicators for the organization of palliative care: A modified RAND Delphi study in seven European countries (the Europall project). Palliat Med 2014;28:121-129.

24. Eagar K, Watters P, Currow D, et al.: The Australian Palliative Care Outcomes Collaboration (PCOC)-Measuring the quality and outcomes of palliative care on a routine basis. Aust Health Rev 2010;34:186-192.

25. Chen J, Ou L, Hollis S: A systematic review of the impact of routine collection of patient reported outcome measures on patients, providers and health organisations in an oncologic setting. BMC Health Serv Res 2013;13:1-24.

26. Luckett T, Butow $\mathrm{P}$, King M: Improving patient outcomes through the routine use of patient-reported data in cancer clinics: Future directions. Psychooncology 2009;18:1129-1138.

27. Howell D, Molloy S, Wilkinson K, et al.: Patient-reported outcomes in routine cancer clinical practice: A scoping review of use, impact on health outcomes, and implementation factors. Ann Oncol 2015;26(9):1846-1858.

28. Etkind S, Daveson B, Kwok W, et al.: Capture, transfer, and feedback of patient-centered outcomes data in palliative care populations: Does it make a difference? A systematic review. J Pain Symptom Manage 2015;49:611-624.

29. Seow H, Sussman J, Martelli-Reid L, et al.: Do high symptom scores trigger clinical actions? An audit after implementing electronic symptom screening. J Oncol Pract 2012;8:e142-e148.

30. Boyce M, Brown J: Does providing feedback on patientreported outcomes to healthcare professionals result in better outcomes for patients? A systematic review. Qual Life Res 2013;22:2265-2278.

31. Catania G, Beccaro M, Costantini M, et al.: Effectiveness of complex interventions focused on quality-of-life assessment to improve palliative care patients' outcomes: A systematic review. Palliat Med 2015;29:5-21.

32. Marshall S, Haywood K, Fitzpatrick R: Impact of patientreported outcome measures on routine practice: A structured review. J Eval Clin Pract 2006;12:559-568.

33. Valderas J, Kotzeva A, Espallargues M, et al.: The impact of measuring patient-reported outcomes in clinical practice: A systematic review of the literature. Qual Life Res 2008; 17:179-193.
34. Kamal A, Bull J, Stinson C, et al.: Conformance with supportive care quality measures is associated with better quality of life in patients with cancer receiving palliative care. J Oncol Pract 2013;9:e73-e76.

35. Abernethy A, Ahmad A, Zafar SY, et al.: Electronic patient-reported data capture as a foundation of rapid learning cancer care. Med Care 2010;48(Suppl 1):S32-S38.

36. Iliffe S, Davies N, Manthorpe J, et al.: Improving palliative care in selected settings in England using quality indicators: A realist evaluation. BMC Palliat Care 2016;15:1-9.

37. Barbera L, Seow H, Sutradhar R, et al.: Quality of end-oflife cancer care in Canada: A retrospective four-province study using administrative health care data. Curr Oncol 2015;22:341-355.

38. Currow D, Eagar K, Aoun S, et al.: Is it feasible and desirable to collect voluntarily quality and outcome data nationally in palliative oncology care? J Clin Oncol 2008;26: 3853-3859.

39. Currow D, Allingham S, Yates $P$, et al.: Improving national hospice/palliative care service symptom outcomes systematically through point-of-care data collection, structured feedback and benchmarking. Support Care Cancer 2015;23:307-315.

40. The Special Senate Committee on Euthanasia and Assisted Suicide. Of Life and Death-Final Report (Internet). Ottawa: The Senate of Canada; 1995. Available from https:// sencanada.ca/content/sen/committee/351/euth/rep/lade.htm (last accessed October 11, 2017).

41. Gaudette LA, Shi F, Lipskie T, et al.: Developing palliative care surveillance in Canada: Results of a pilot study. $\mathrm{J}$ Palliat Care 2002;18:262-269.

42. Lau F, Downing M, Tayler C, et al.: Toward a populationbased approach to end-of-life care surveillance in Canada: Initial efforts and lessons. J Palliat Care 2013;29:13-21.

43. Allan DE, Stajduhar KI, Reid RC: The uses of provincial administrative health databases for research on palliative care: Insights from British Columbia, Canada. BMC Palliat Care 2005;4:2.

44. Gagnon B, Nadeau L, Scott S, et al.: The association between home palliative care services and quality of end-oflife care indicators in the province of Quebec. J Pain Symptom Manage 2015;50:48-58.

45. Tanuseputro P, Budhwani S, Bai YQ, Wodchis W. Palliative care delivery accross health sectors: A population-level observational study. Palliat Med 2017;31(3):247-257.

46. Seow H, Pataky R, Lawson B, et al.: Temporal association between home nursing and hospital costs at the end of life in three provinces. Curr Oncol 2016;23(Suppl 1):S42-S51.

47. Dudevich A, Chen A, Gula C, et al.: End-of-life hospital care for cancer patients: An update. Age 2014;20(44):8-10.

48. Canadian Partnership Against Cancer: The 2016 Cancer System Performance Report. Toronto, Ontario, p.128.

49. Accreditation Agreement Canada Qmentum: Hospice, Palliative and End-of-Life Services. Report No.: Version 7, 2016.

Address correspondence to: Deborah Dudgeon, MD, FRCPC School of Medicine Queen's University 34 Barrie Street Kingston, Ontario K7L $3 J 7$ Canada

E-mail: dudgeond@kgh.kari.net 